\title{
Magnetic properties, exchange bias, and memory effects in core-shell superparamagnetic nanoparticles of $\mathrm{La}_{0.67} \mathrm{Sr}_{0.33} \mathrm{MnO}_{3}$
}

A. Rostamnejadi, M. Venkatesan, H. Salamati, K. Ackland, H. Gholizadeh, P. Kameli, and J. M. D. Coey

Citation: Journal of Applied Physics 121, 173902 (2017);

View online: https://doi.org/10.1063/1.4982893

View Table of Contents: http://aip.scitation.org/toc/jap/121/17

Published by the American Institute of Physics

\section{Articles you may be interested in}

Size-induced changes of structural and ferromagnetic properties in $\mathrm{La}_{1-x} \mathrm{Sr}_{x} \mathrm{MnO}_{3}$ nanoparticles

Journal of Applied Physics 121, 214303 (2017); 10.1063/1.4984829

Growth and ferroelectric properties of $\mathrm{La}$ and $\mathrm{Al}$ codoped $\mathrm{BiFeO}_{3}$ epitaxial films

Journal of Applied Physics 121, 174102 (2017); 10.1063/1.4982909

Giant perpendicular exchange bias with antiferromagnetic $\mathrm{MnN}$

Applied Physics Letters 110, 192402 (2017); 10.1063/1.4983089

Giant-spin nonlinear response theory of magnetic nanoparticle hyperthermia: A field dependence study Journal of Applied Physics 121, 173901 (2017); 10.1063/1.4982357

Room-temperature five-tesla coercivity of a rare-earth-free shell-ferromagnet

Applied Physics Letters 110, 192406 (2017); 10.1063/1.4983199

Spin pumping torque in antiferromagnets

Applied Physics Letters 110, 192405 (2017); 10.1063/1.4983196

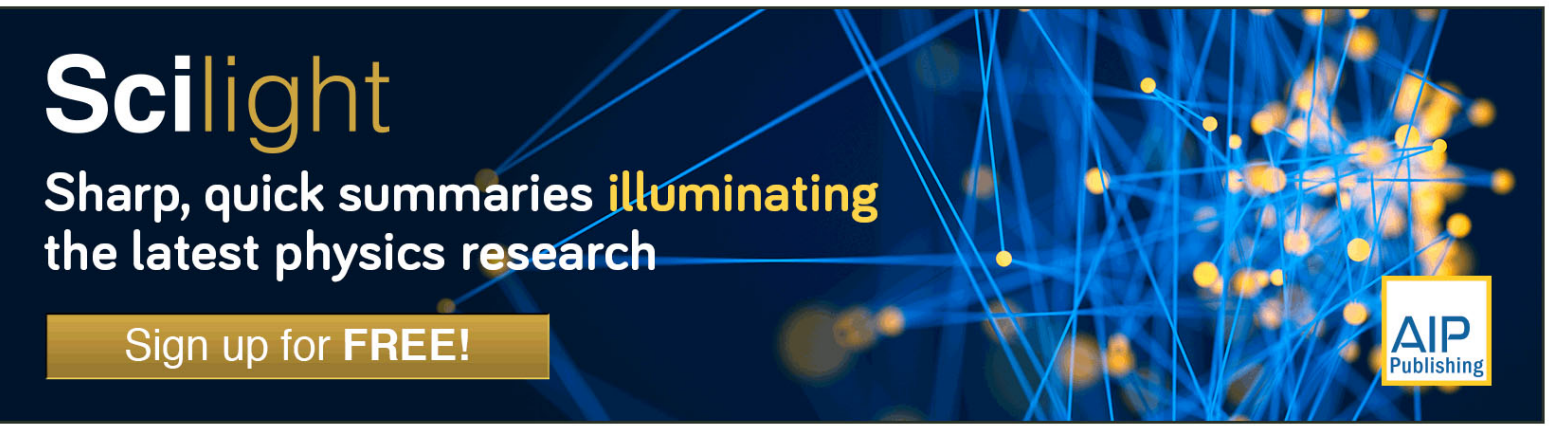




\title{
Magnetic properties, exchange bias, and memory effects in core-shell superparamagnetic nanoparticles of $\mathrm{La}_{0.67} \mathrm{Sr}_{0.33} \mathrm{MnO}_{3}$
}

\author{
A. Rostamnejadi, ${ }^{1,2, a)}$ M. Venkatesan, ${ }^{3} \mathrm{H}$. Salamati, ${ }^{2} \mathrm{~K}$. Ackland, ${ }^{3} \mathrm{H}$. Gholizadeh, ${ }^{2}$ \\ P. Kameli, ${ }^{2}$ and J. M. D. Coey ${ }^{3}$ \\ ${ }^{1}$ Electroceram Research Centre, Malek Ashtar University of Technology, Iran \\ ${ }^{2}$ Department of Physics, Isfahan University of Technology, Isfahan, Iran \\ ${ }^{3}$ School of Physics, Trinity College, Dublin 2, Ireland
}

(Received 24 January 2017; accepted 20 April 2017; published online 4 May 2017)

\begin{abstract}
The static magnetic properties and memory and exchange bias effects have been studied in sol-gel prepared $\mathrm{La}_{0.67} \mathrm{Sr}_{0.33} \mathrm{MnO}_{3}$ (LSMO) nanoparticles. Transmission electron microscopy (TEM) micrographs and static magnetization show log-normal particle and magnetic size distributions with a core-shell structure. Analysis of the magnetization measurements indicates the presence of a magnetic structure with a $7.8 \mathrm{~nm}$ core radius and a magnetic dead layer of thickness $1.6 \mathrm{~nm}$ in the LSMO nanoparticles, which comprises about $40 \%$ of the volume. The disordered spins in the shell freeze at lower temperatures than the core and produce a surface spin glass state exhibiting a weak exchange bias effect. Field cooled and zero-field cooled magnetization measurements have been carried out to study the slow dynamics of the sample and associated magnetic memory effects; the results reveal the superparamagnetic behavior of LSMO nanoparticles described in terms of the magnetic size distribution rather than a superspin glass state. Published by AIP Publishing.

[http://dx.doi.org/10.1063/1.4982893]
\end{abstract}

\section{INTRODUCTION}

Magnetic nanostructures have been extensively studied due to their interesting fundamental physics and their technological and medical applications. ${ }^{1-4}$ In general, the magnetic properties of nanoparticles are strongly influenced by changing their size. The static and dynamic magnetic response of a small single-domain magnetic nanoparticle can be considered equivalent to the response of a single large spin, called a macrospin or superspin. ${ }^{5-9}$ While non-interacting superspins show superparamagnetic behavior, strong interparticle interactions can lead to a superspin glass state in a dense ensemble of magnetic nanoparticles. ${ }^{5-7,9-13}$ Collective freezing of the superspins can be studied by various static and dynamic magnetic techniques. Measurements such as frequency-dependent AC magnetic susceptibility, field cooled (FC) and zero-field cooled (ZFC) magnetization as a function of temperature, and time-dependent magnetization to investigate aging, memory effects, and non-exponential relaxation are used to study the slow dynamical properties of superparamagnetic and spin glass systems. ${ }^{5,9-12,14-16}$

The $\mathrm{La}_{1-\mathrm{x}} \mathrm{Sr}_{\mathrm{x}} \mathrm{MnO}_{3}$ perovskite manganites are some of the most captivating magnetic materials due to their colossal magnetoresistance, half-metallicity, and magnetocaloric and optical properties. ${ }^{4,17,18}$ Among these manganites, $\mathrm{La}_{1-\mathrm{x}} \mathrm{Sr}_{\mathrm{x}} \mathrm{MnO}_{3}$ with $\mathrm{x}=0.33$ has the highest Curie temperature of $380 \mathrm{~K} .{ }^{18}$ Single-domain magnetic nanoparticles with large saturation magnetization and a Curie temperature above room temperature have potential for use in hyperthermia, MRI contrast enhancement, and drug delivery applications. It is therefore pertinent to study the static and dynamic

\footnotetext{
${ }^{\text {a) }}$ Author to whom correspondence should be addressed. Electronic mail: ali@ph.iut.ac.ir
}

magnetic properties of the optimally doped manganite. We have previously examined the dynamic magnetic properties of $\mathrm{La}_{0.67} \mathrm{Sr}_{0.33} \mathrm{MnO}_{3}$ (LSMO) nanoparticles by $\mathrm{AC}$ magnetic susceptibility measurements; ${ }^{13}$ the results indicated that the nanoparticles have an interacting superparamagnetic behavior above room temperature and are of interest for medical applications. ${ }^{19-22}$

In the present research, we have studied the static magnetic properties, exchange bias, and memory effects. TEM and HRTEM micrographs and static magnetization measurements indicate particle and magnetic size distributions possessing a magnetic core-shell structure. FC and ZFC memory experiments have been performed by measuring the magnetization as a function of temperature. The results confirm the weakly interacting superparamagnetic character of the LSMO nanoparticles.

\section{EXPERIMENT}

Single-phase nanoparticles of $\mathrm{La}_{0.67} \mathrm{Sr}_{0.33} \mathrm{MnO}_{3}$ (LSMO) have been synthesized by a sol-gel method. The details of the preparation method are given elsewhere. ${ }^{13}$ The particle size distribution of the sample is estimated from TEM micrographs, using a FEI Titan field-emission TEM operating at an accelerating voltage of $300 \mathrm{kV}$. Static and dynamic magnetic properties have been studied using a SQUID magnetometer (Quantum Design MPMS) in the range of $\pm 5 \mathrm{~T}, 5-400 \mathrm{~K}$.

\section{RESULTS AND DISCUSSION}

The crystal structure of the nanoparticles has been characterized by X-ray diffraction and analyzed using the Rietveld method; it is found that the particles have a rhombohedral crystal structure with space group $R \overline{3} C .{ }^{13}$ The TEM 


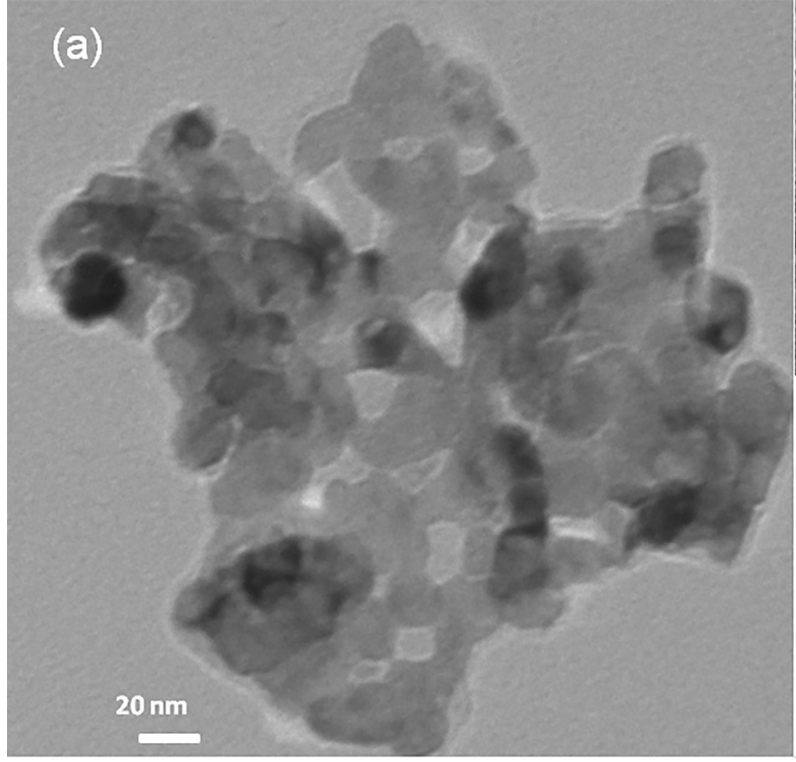

FIG. 1. (a) TEM micrograph of LSMO nanoparticles. (b) HRTEM image of a nanoparticle. (c) The particle size distribution obtained from the TEM images and the fit with a log-normal function. micrograph in Fig. 1(a) shows that the particles possess a quasi-spherical morphology. The HRTEM micrograph in Fig. 1(b) confirms the crystalline nature of the LSMO nanoparticles. The particle size distribution has been deduced from the TEM micrograph, and it fits rather well with a lognormal function as shown in Fig. 1(c). The log-normal function is given as ${ }^{23,24}$

$$
\mathrm{f}(\mathrm{d})=\frac{1}{w d \sqrt{2 \pi}} \exp \left[-\frac{\left(\ln \left(d / d_{\text {med }}\right)\right)^{2}}{2 w^{2}}\right] .
$$

In this relation, $d$ and $d_{\text {med }}$ are the particle size and its median value, respectively, while $w$ is the dimensionless standard deviation. The median value of the particle size and its deviation obtained from the log-normal fitting are $19.8 \mathrm{~nm}$ and 0.24 , respectively.

The dynamic magnetic properties of the sample presented here have been reported in detail in a previous paper. ${ }^{13}$ Analysis of the AC magnetic susceptibility using Vogel-Fulcher and critical slowing-down models suggests an interacting superparamagnetic behavior near room temperature with an effective magnetic anisotropy constant $K_{\text {eff }}=2.25 \times 10^{4} \mathrm{~kJ} / \mathrm{m}^{3} .{ }^{13}$ SQUID magnetometry is used here to measure the detailed field and temperature dependence of the magnetization. FC and ZFC magnetization analyses give useful information about the irreversibility of the magnetization; these techniques will be used to distinguish between superparamagnetic behavior and a superspin glass state. The temperature dependence of the magnetization is depicted in FC and ZFC modes for applied magnetic fields of $\mu_{0} H=1,5$, and $50 \mathrm{mT}$ in Fig. 2; the ZFC and FC magnetization curves bifurcate upon decreasing temperature at $T_{\mathrm{B}}$, which indicates the onset of a blocking/freezing process. It can also be seen that $T_{\mathrm{B}}$ is lowered by increasing the applied magnetic field, which may be explained as follows; by applying an external magnetic field, the potential barrier due to magnetic anisotropy is reduced and thermal energy overcomes the barrier. It is clear from Fig. 2 that the FC magnetization of the sample increases with decreasing temperature below $T_{\mathrm{B}}$, a typical feature of superparamagnetic systems. ${ }^{6}$ In this figure, the Zeeman interaction energy is the main type of interaction between magnetic nanoparticles in an applied magnetic field. When the field strength is sufficiently small, the Zeeman energy cannot influence the spin (exchange and dipolar) interactions and the FC magnetization as a function of temperature is flattened. There is also a kink evident in the ZFC magnetization at $\sim 175 \mathrm{~K}$, which may be due to spin freezing in the surface layer of the nanoparticles. ${ }^{25-28}$ The observed magnetic properties of LSMO nanoparticles are often described in terms of competing double exchange and superexchange interactions. Double exchange usually mediates the ferromagnetic state, while superexchange usually mediates an antiferromagnetic state in magnetic oxides. ${ }^{4,17,29}$ At the surface of magnetic nanoparticles, there are broken and incomplete chemical bonds that can randomly change the balance of ferromagnetic and antiferromagnetic exchange interactions, thereby providing randomness and frustration for the surface spin glass. In the presence of the $50 \mathrm{mT}$ applied field, which is larger in magnitude than the low temperature coercive field of the sample, the Zeeman

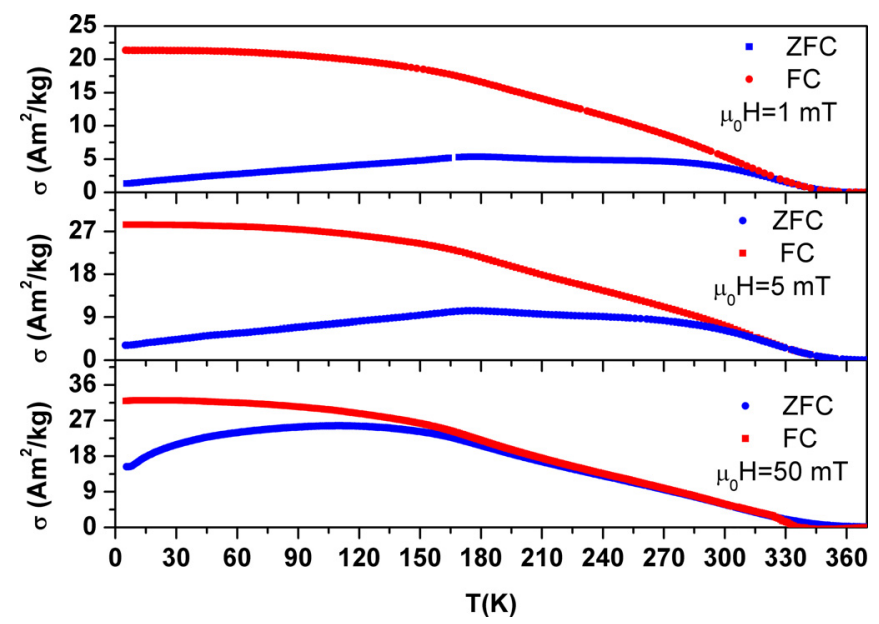

FIG. 2. FC and ZFC magnetization of LSMO nanoparticles as a function of temperature at applied magnetic fields of 1,5 , and $50 \mathrm{mT}$. 
energy overcomes the interaction of the spins with their surroundings and the kink disappears. Hence, ZFC and FC magnetization measurements confirm the superparamagnetic behavior of the LSMO nanoparticles, consistent with the AC magnetic susceptibility results presented previously. ${ }^{13}$ The bifurcations in Fig. 2 do not simply reflect the temperaturedependence of the coercive field since they occur at temperatures where $H_{\mathrm{c}} \ll H_{\mathrm{a}}$.

Field-dependent magnetization at different temperatures is plotted in Fig. 3; it can be seen that at $330 \mathrm{~K}$ and $300 \mathrm{~K}$, the coercive field and remanence are zero, which establishes the superparamagnetic nature of the sample above room temperature. In the superparamagnetic regime, the magnetization $\sigma_{S P M}$ of the sample as a function of applied field $H$ can be expressed as an integrated log-normal weighted Langevin function of the following form: ${ }^{23,24}$

$$
\sigma_{S P M}(H)=N \int_{0}^{\infty} \mu f(\mu) \mathcal{L}\left(\mu_{0} \mu \mathrm{H} / k_{B} \mathrm{~T}\right) \mathrm{d} \mu+\chi H,
$$

where $\mu$ is the magnetic moment of a nanoparticle, $f(\mu)$ is the log-normal function given previously in Equation (1), $N$ is the particle number per unit of mass, $\chi$ is the high-field susceptibility, and $\mathcal{L}(\mathrm{x})$ is the Langevin function, where $x=\mu_{0} \mu \mathrm{H} /$ $k_{B} \mathrm{~T}$ and $\mathcal{L}(\mathrm{x})=\operatorname{coth}(\mathrm{x})-1 / \mathrm{x}$. The magnetic moment distribution width $w_{\text {mag }}$ and mean magnetic moment $\mu_{\text {mean }}$ are related to the median magnetic moment distribution $\mu_{\text {med }}$

$$
\mu_{\text {mean }}=\mu_{\text {med }} \exp \left(w_{\text {mag }}^{2} / 2\right) .
$$

The saturation magnetization $\sigma_{S}$ is given by the following relation:

$$
\sigma_{S}=N \int_{0}^{\infty} \mu f(\mu) \mathrm{d} \mu=\frac{N}{w_{\text {mag }} \sqrt{2 \pi}} \int_{0}^{\infty} \exp \left[-\frac{\left(\ln \left(\mu / \mu_{\text {med }}\right)\right)^{2}}{2 w_{\text {mag }}^{2}}\right] d \mu .
$$

The magnetic moment distribution of the sample at $330 \mathrm{~K}$ and $300 \mathrm{~K}$ has been obtained from the experimental (a)
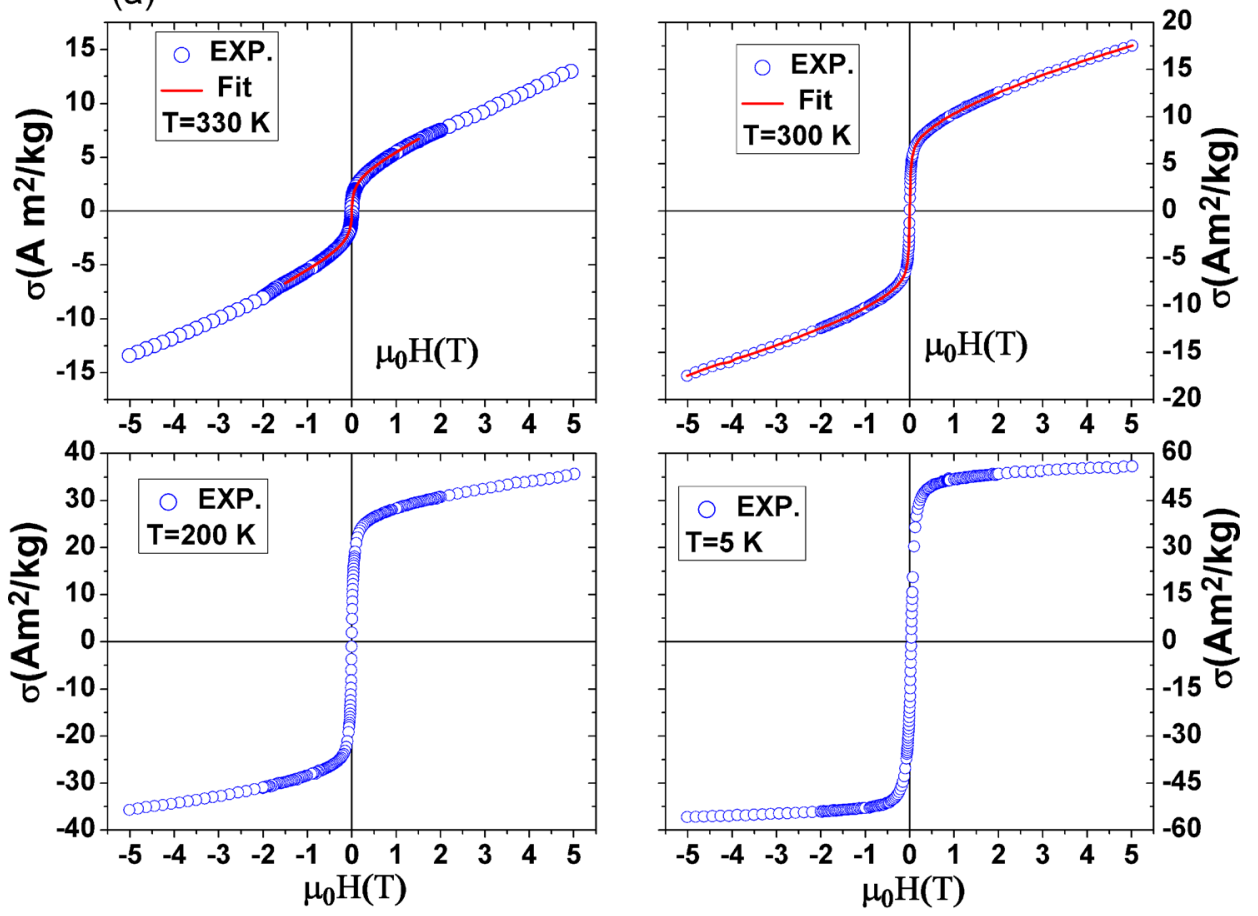

(b)

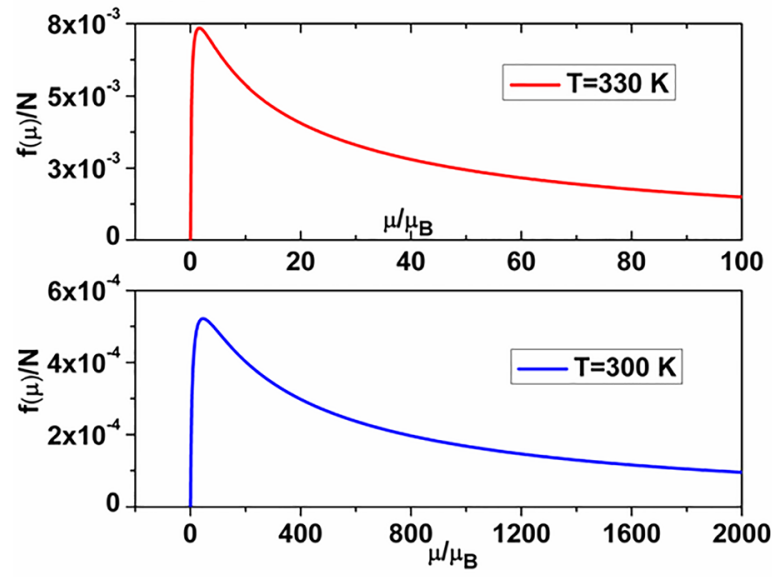

(c)

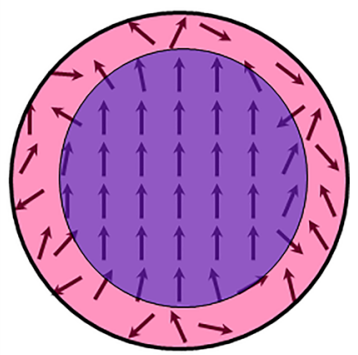

FIG. 3. (a) Magnetization of LSMO nanoparticles as a function of the applied magnetic field at different temperatures. The solid red lines show the log-normal weighted Langevin function fit with the experimental data. (b) Magnetic size distribution obtained from the log-normal distribution function of $\sigma-H$ curves at $T=330$ and 300 K. (c) Suggested magnetic coreshell structure for a nanoparticle. 
magnetization-field data using Equation (2). The fitted curves and their corresponding magnetic moment distributions are depicted in Fig. 3(a). At $T=300 \mathrm{~K}$ and $330 \mathrm{~K}$, the values obtained for $\sigma_{S}$ are $9.59 \mathrm{Am}^{2} / \mathrm{kg}$ and $4.25 \mathrm{Am}^{2} / \mathrm{kg}$, for $N$ are $3.3 \times 10^{22} / \mathrm{kg}$ and $9.2 \times 10^{19} / \mathrm{kg}$, for $\mu_{\text {med }}$ are $3.04 \times 10^{3} \mu_{\mathrm{B}}$ and $3.42 \times 10^{2} \mu_{\mathrm{B}}$, for $\chi$ are 1.68 and 2.10 $\left(\mathrm{Am}^{2} / \mathrm{kg}\right) / \mathrm{T}$, and for $w_{\text {mag }}$ (magnetic moment distribution width) are 2.05 and 2.32, respectively. These results show that there is a magnetic moment distribution in the LSMO nanoparticles due to the particle size distribution.

In $\mathrm{La}_{1-x} \mathrm{Sr}_{x} \mathrm{MnO}_{3}$, there are $\mathrm{Mn}^{3+}(\mathrm{S}=2)$ and $\mathrm{Mn}^{4+}$ $(\mathrm{S}=3 / 2)$ ions in proportions $1-\mathrm{x}$ and $\mathrm{x}$, respectively. ${ }^{17}$ The average spin-only magnetic moment of $\mathrm{Mn}$ ions in the ferromagnetic state can be calculated as

$$
\mu_{t h}=4(1-x) \mu_{B}+3 x \mu_{B}=(4-x) \mu_{B},
$$

where $\mu_{B}$ is the Bohr magneton. In our case, the theoretical value of the magnetic moment is $3.67 \mu_{\mathrm{B}} / \mathrm{Mn}$. Experimentally, the spontaneous magnetic moment of the nanoparticles can be estimated from the linear part of the $M-H$ curve at high magnetic fields as

$$
m(T, H)=m_{s p}(T)+\chi H .
$$

In this relation, $m_{s p}$ is the spontaneous magnetization and $\chi$ is the high-field susceptibility. Knowing the molar mass of the compound, the spin-only magnetic moment in $\mu_{B}$ can be obtained as ${ }^{30}$

$$
m\left(\mu_{B / \text { formula unit }}\right)=\frac{\sigma \mathrm{M}}{1000 N_{A} \mu_{\mathrm{B}}} .
$$

In this relation, $M$ is the molar mass ( $\mathrm{g} / \mathrm{mole}), \sigma$ is the magnetization $\left(\mathrm{Am}^{2} / \mathrm{kg}\right)$, and $N_{A}$ is the Avogadro number $(6.022$ $\times 10^{23}$ ). The spontaneous magnetic moment of $\mathrm{Mn}$ ions in LSMO nanoparticles at $T=5,200,300$, and $330 \mathrm{~K}$ is $m_{\mathrm{sp}}=2.10,1.10,0.36$, and $0.16 \mu_{\mathrm{B}}$, respectively. The ferromagnetic phase fraction in a LSMO nanoparticle can be calculated as

$$
\frac{V_{F M}}{V_{\text {particle }}}=\frac{m_{s p}}{3.67}
$$

At $T=5 \mathrm{~K}$, the ferromagnetic phase fraction is about $57 \%$ of the theoretical value. We can estimate the low-temperature ferromagnetic core radius and the disordered shell thickness as shown in Fig. 3(c). Using the mean particle size obtained from the TEM micrograph and assuming a spherical shape for nanoparticles, we obtain $7.8 \mathrm{~nm}$ and $1.6 \mathrm{~nm}$ for the core radius and thickness of the dead magnetic shell, respectively.

Due to the possible interface exchange interactions between disordered frozen spins in the shell and the ferromagnetic core, an exchange bias effect could be expected in LSMO nanoparticles; to this end, we have measured fieldcooled hysteresis loops for $0,1,10$, and $100 \mathrm{mT}$ at $4 \mathrm{~K}$, which are shown in Fig. 4. From this figure, it is seen that virtually symmetric hysteresis loops are measured, with a small loop shift in the opposite direction to the cooling field. In exchange bias systems, the coercive field and the exchange bias field are defined as

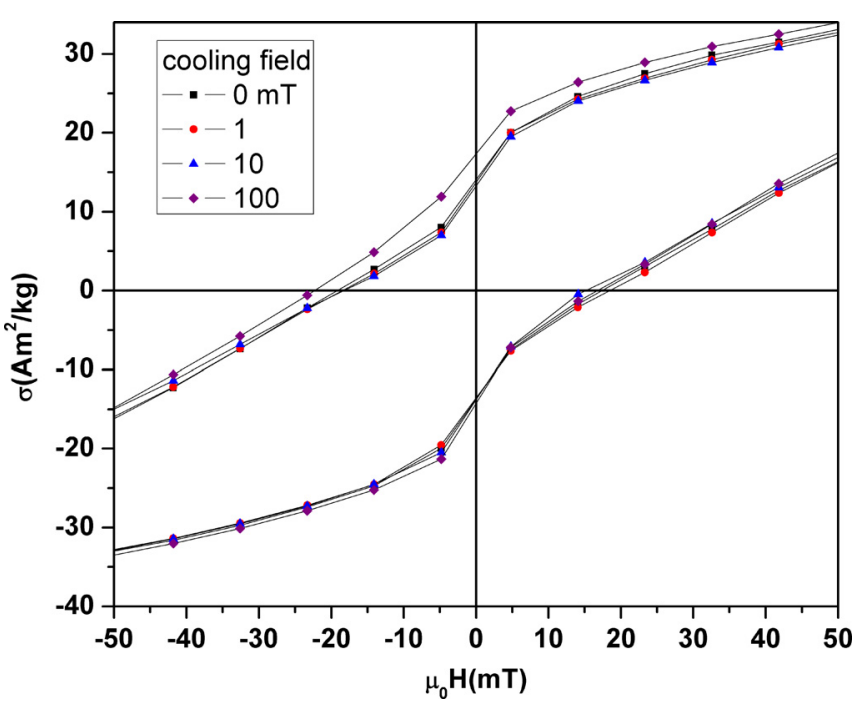

FIG. 4. Magnetic hysteresis loops of LSMO nanoparticles at $4 \mathrm{~K}$ in cooling fields of $0,1,10$, and $100 \mathrm{mT}$.

$$
H_{\mathrm{c}}=-\left(H_{1}-H_{2}\right) / 2 \quad \text { and } \quad H_{\mathrm{eb}}=-\left(H_{1}+H_{2}\right) / 2,
$$

where $H_{1}$ and $H_{2}$ are the negative and positive coercive fields, respectively. By increasing the cooling field from 0 to $100 \mathrm{mT}$, the coercive field $\left(\mu_{0} H_{\mathrm{c}}\right)$ increases from $18.2 \mathrm{mT}$ to $19.6 \mathrm{mT}$ and the exchange bias field $\left(\mu_{0} H_{\mathrm{eb}}\right)$ exceeds $2.7 \mathrm{mT}$. The exchange bias field in a cooling field of $5 \mathrm{~T}$ at $5 \mathrm{~K}$ is also around $3 \mathrm{mT}$. Weak exchange coupling between ferromagnetic core spins and the frozen spins in the disordered shell layer leads to a small interfacial exchange energy. Because of the relatively large ferromagnetic thickness and the strong cooling magnetic field, the Zeeman energy is much larger in magnitude than the interfacial exchange energy; therefore, the exchange bias effect is weak. This is similar to the ferromagnetic/antiferromagnetic bilayer systems, for which upon increasing the ferromagnetic thickness, the exchange bias field decreases and eventually reduces to zero for relatively large thicknesses. ${ }^{31}$

There are some common features among superparamagnetic and superspin glass systems. There is a characteristic frequency-dependent peak in the AC magnetic susceptibility in both the cases. The frequency dependence of the blocking/ freezing temperature may be evaluated by Néel-brown, Vogel-Fulcher, and critical slowing-down models, but they are difficult to distinguish. The FC and ZFC magnetization as a function of temperature also has a common behavior in both the systems. The slow magnetization dynamics is similar to the FC magnetization response. To study the slow dynamical properties of the sample, we have employed three different measurement protocols in the FC and ZFC magnetization measurements, which we describe in detail in the next paragraphs.

In the first protocol, we cool the sample from room temperature to $5 \mathrm{~K}$ in a constant applied field of $5 \mathrm{mT}$. Then, the magnetization is measured during heating from $5 \mathrm{~K}$ to room temperature in the presence of the applied magnetic field. The magnetization curve obtained is depicted by the solid line in Fig. 5, denoted as "reference." The sample is then cooled from room temperature in a $5 \mathrm{mT}$ magnetic field, and 


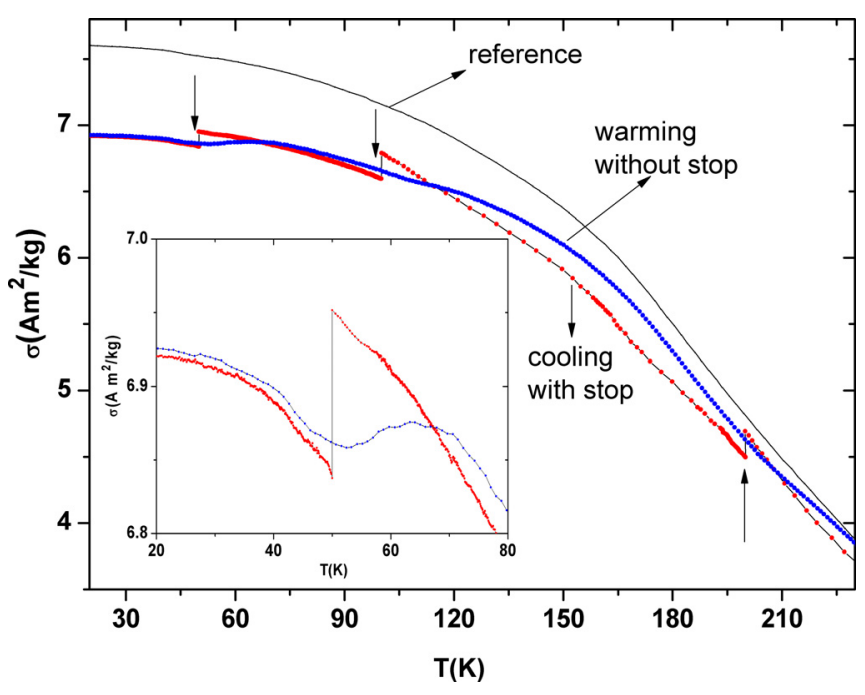

FIG. 5. Field cooled magnetic memory effect of LSMO nanoparticles. The inset shows the low temperature region on an expanded magnetization scale.

the magnetization is measured as a function of temperature, remaining at $200 \mathrm{~K}$ for $1 \mathrm{~h}$. Then, the applied magnetic field is removed during the waiting period to allow the magnetic relaxation of the nanoparticles. After this $1 \mathrm{~h}$ waiting period, the magnetic field is re-applied and the magnetization of the sample is measured from $200 \mathrm{~K}$ down to $100 \mathrm{~K}$. The applied magnetic field is again removed for a period of $1 \mathrm{~h}$. The same process is repeated at $\mathrm{T}=100$ and $50 \mathrm{~K}$. The measured magnetization in this cooling procedure gives the step-like curve, which is shown in Fig. 5. After reaching $5 \mathrm{~K}$, the temperature is increased continuously at a heating rate of $1 \mathrm{~K} /$ min and the sample magnetization is measured during the heating process in the constant $5 \mathrm{mT}$ magnetic field. The measured magnetization in the cooling process showed distinct step-like behavior at the stopping temperatures. On increasing the temperature again, the magnetization has an upturn around the stopping temperature at $50 \mathrm{~K}$ and then reaches its value in the cooling process only a few kelvins above it. Similar behavior, but with a less change in the magnetization value, has been observed at the other stopping temperatures $\mathrm{T}=100$ and $200 \mathrm{~K}$. The measured magnetization in this protocol shows that LSMO nanoparticles remember their thermal history at the stopping temperatures during the cooling.

In the second measurement protocol, we have measured the magnetization in a similar way to the first protocol. First, we measured the reference magnetization in the FC mode from $5 \mathrm{~K}$ to room temperature. Then, the sample is cooled down from room temperature to $5 \mathrm{~K}$ in a $5 \mathrm{mT}$ constant magnetic field. At a stopping temperature of $50 \mathrm{~K}$, the magnetic field is removed for $0.1 \mathrm{~h}$ to let the magnetization relax. Then, while the magnetization is being monitored, the sample temperature is reduced from $50 \mathrm{~K}$ to $40 \mathrm{~K}$, then increased from $40 \mathrm{~K}$ to $55 \mathrm{~K}$, and subsequently again decreased from $55 \mathrm{~K}$ to $50 \mathrm{~K}$ in the $5 \mathrm{mT}$ magnetic field. This process has been repeated for waiting times of $0.2,0.5$, and $1 \mathrm{~h}$ at $50 \mathrm{~K}$. The measured magnetization, which is shown in Fig. 6, shows again the step-like trend similar to that observed in the first protocol. In each period, the measured magnetization

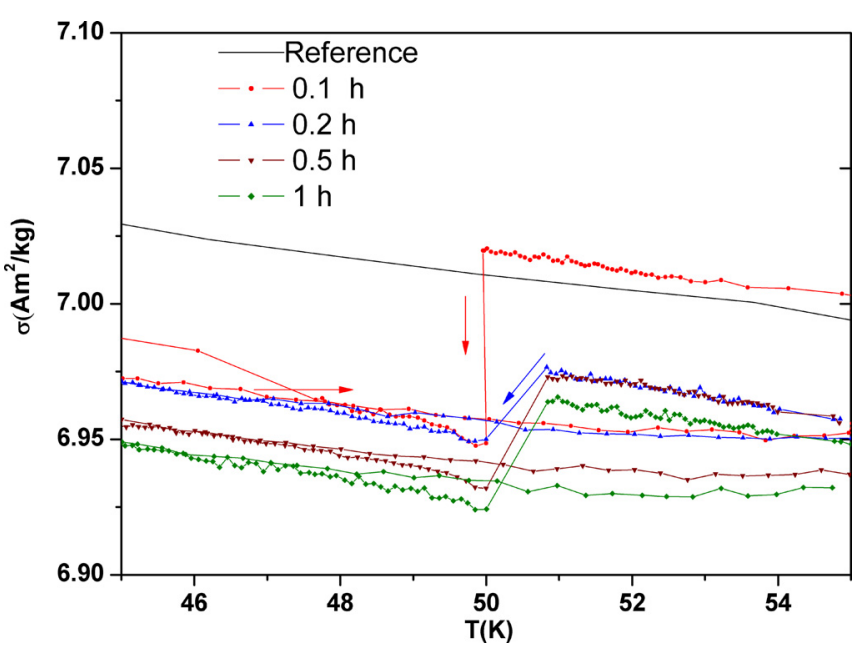

FIG. 6. FC memory effect of LSMO nanoparticles with different waiting times at $50 \mathrm{~K}$.

in the heating stage has the tendency to return to its value in the cooling process a few kelvins above the stopping temperature. This tendency is reduced by increasing the waiting periods. It can also be seen from Fig. 6 that the difference in the measured magnetization between the cooling and heating processes is reduced by repeating the waiting time process at $50 \mathrm{~K}$.

Memory effects should weaken with decreasing interparticle interactions. ${ }^{5,12,32,33}$ To investigate their influence on the FC memory effect, LSMO nanoparticles have been diluted by mixing them with icing sugar with a $1.3 / 30.6 \mathrm{mg}$ weight ratio. Then, the second protocol has been employed on the LSMO/icing sugar mixture with different waiting times of $0.1,0.2,0.3,1$, and $2 \mathrm{~h}$ at $50 \mathrm{~K}$. The measured magnetization during the different waiting periods is shown in Fig. 7, where it is evident that the difference in the measured magnetization between the cooling and heating processes in the first period is larger than those for the successive periods, and the difference becomes negligible after several

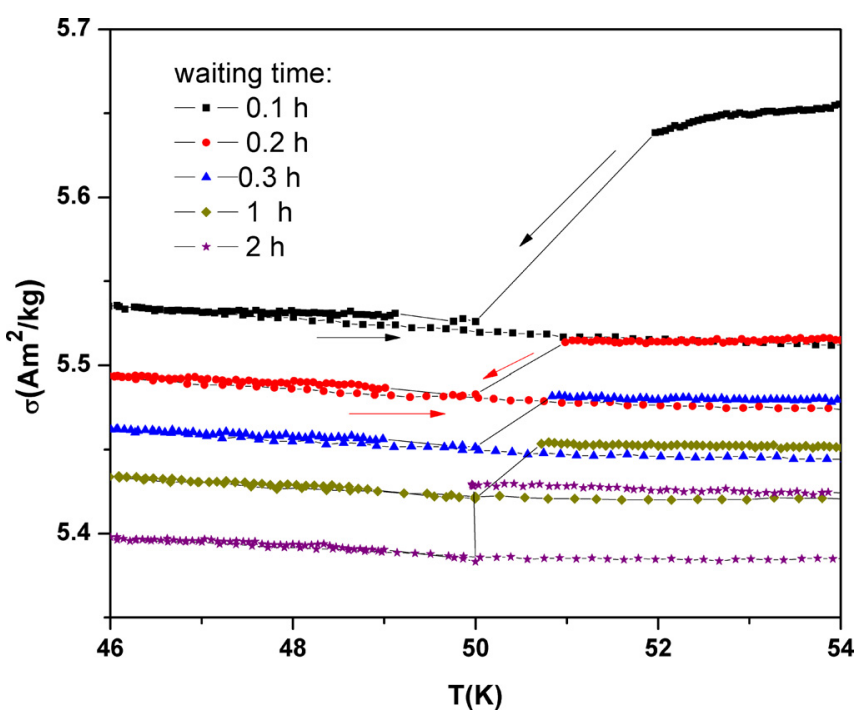

FIG. 7. FC memory effect of dilute LSMO/icing sugar nanoparticle samples with different waiting times at $50 \mathrm{~K}$. 
successive waiting periods. The magnetization measured during the heating process has the tendency not to return to its value measured during the cooling process. In effect, in the LSMO/icing sugar mixture, the FC memory effect is weakened by decreasing the interparticle interaction. It suggests that the interparticle interaction is one of the main origins for the slow dynamics observed in LSMO nanoparticles.

In the third measurement protocol, we have used the ZFC magnetic memory effect. In this procedure, the sample is cooled down from room temperature to $5 \mathrm{~K}$ in the absence of a magnetic field, and then the magnetization is measured with increasing temperature from $5 \mathrm{~K}$ to room temperature in a constant $5 \mathrm{mT}$ field, which is the reference curve for the ZFC memory effect, shown by a solid line in Fig. 8. In the second stage of this protocol, the sample is cooled down from room temperature to stopping temperatures at $50 \mathrm{~K}$ and $10 \mathrm{~K}$ in the absence of the external magnetic field. It is held at the stopping temperatures of 50 and $10 \mathrm{~K}$ for $1 \mathrm{~h}$. Subsequently, the sample is cooled down to $5 \mathrm{~K}$, then a $5 \mathrm{mT}$ magnetic field is applied, and the magnetization is recorded during heating from $5 \mathrm{~K}$ up to room temperature. The measured magnetization is depicted in Fig. 8. As can be seen from Fig. 8, no significant drop has been found at the stopping temperatures relative to the reference ZFC magnetization curve. If the system was in a superspin glass state, a large drop in the ZFC magnetization at the stopping points would be expected. Hence, the ZFC memory effect suggests superparamagnetic behavior in LSMO nanoparticles rather than a superspin glassy state.

Three possible mechanisms could be considered to describe the slow dynamics in magnetic nanostructures. ${ }^{5-7}$ The first mechanism originates from the broad distribution of the relaxation times of the nanoparticles, which is due to the magnetic size distribution or the distribution in effective anisotropy energy barriers of the nanoparticles. This is the main source of slow dynamics in the coherent state of single domain non-interacting particles. Disordered interacting surface spins or surface ferromagnetic clusters can also mediate the surface spin glass state, which can be considered as the second source of slow dynamics in magnetic nanoparticles.

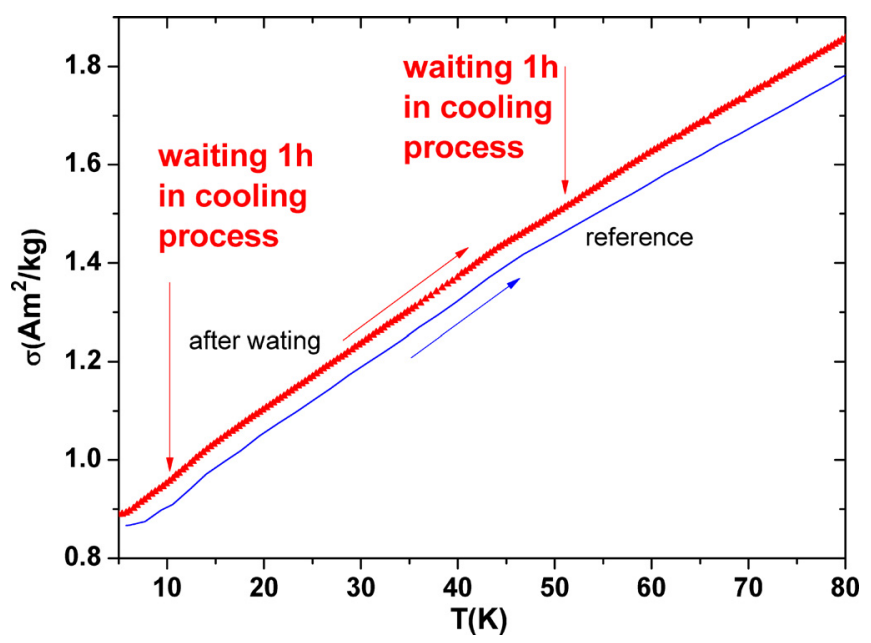

FIG. 8. Absence of the zero field cooled magnetic memory effect of LSMO nanoparticles.
The third mechanism of slow dynamics in magnetic nanoparticles originates from the strong interparticle interaction. In the same manner as in atomic spin glass systems, the strong dipole-dipole magnetic interactions could also mediate a superspin glass state in a dense ensemble of magnetic nanoparticles. Frustration and randomness in the position, effective magnetic anisotropy, and magnetization direction of the nanoparticles may induce a cooperative spin glass state in a dense ensemble of magnetic nanoparticles.

The FC memory effect has been observed in both the superparamagnetic and spin glass systems. ${ }^{5,32-34}$ The ZFC memory effect has only been measured in spin glass systems. ${ }^{5,33}$ The FC memory effect observed in LSMO nanoparticles is mainly due to the magnetic size distribution, which we have observed in the log-normal distribution of the magnetic moment in the $\mathrm{M}-\mathrm{H}$ measurements above the blocking temperature. The surface spin glass state may also contribute to the FC memory effect, but it is too weak to be seen in the ZFC memory effect. Therefore, the observed FC and ZFC memory effect results indicate a superparamagnetic behavior in LSMO nanoparticles, consistent with the results obtained from AC magnetic susceptibility.

\section{CONCLUSIONS}

We have investigated the magnetic properties and memory effects of LSMO nanoparticles to have an intrinsic coreshell magnetic structure with superparamagnetic behavior below room temperature. The particle and magnetic size distributions deduced from the TEM images and M-H measurements are log-normal distribution functions. The ferromagnetic moment of our nanoparticles is only about $60 \%$ of the bulk value, due to a $1.6 \mathrm{~nm}$ shell of disordered frozen spins at the surface. The existence of a kink in the ZFC M-T curves and the weak exchange bias effect support surface spin disorder in the nanoparticles. The observed memory effect in the FC process and its absence in the ZFC process confirm the superparamagnetism of the LSMO nanoparticles rather than a superspin glass behavior.

\section{ACKNOWLEDGMENTS}

This work was supported by Science Foundation Ireland (SFI) as part of the MANSE Project Grant No. SFI05/IN/ 1850. The TEM was enabled by the Advanced Microscopy Laboratory (AML) facility at the AMBER Centre, CRANN Institute, Trinity College Dublin.

${ }^{1}$ A. P. Guimaraes, Principles of Nanomagnetism (Springer-Verlag, Berlin/ Heidelberg, 2009).

${ }^{2}$ S. P. Gubin, Magnetic Nanoparticles (Wiley Online Library, 2009).

${ }^{3}$ R. N. Mahato, K. Sethupathi, V. Sankaranarayanan, and R. Nirmala, J. Appl. Phys. 107, 09A943 (2010).

${ }^{4}$ A. Rostamnejadi, M. Venkatesan, J. Alaria, M. Boese, P. Kameli, H. Salamati, and J. M. D. Coey, J. Appl. Phys. 110, 043905 (2011).

${ }^{5}$ M. Sasaki, P. Jönsson, H. Takayama, and H. Mamiya, Phys. Rev. B 71, 104405 (2005).

${ }^{6}$ D. Parker, F. Ladieu, E. Vincent, G. Mériguet, E. Dubois, V. Dupuis, and R. Perzynski, J. Appl. Phys. 97, 10A502 (2005).

${ }^{7}$ M. Suzuki, S. I. Fullem, I. S. Suzuki, L. Wang, and C. J. Zhong, Phys. Rev. B 79, 24418 (2009).

${ }^{8}$ C. R. Sankar and P. Joy, Phys. Rev. B 72(13), 132407 (2005). 
${ }^{9}$ S. Sahoo, O. Petracic, C. Binek, W. Kleemann, J. Sousa, S. Cardoso, and P. Freitas, Phys. Rev. B 65, 134406 (2002).

${ }^{10}$ S. Bedanta and W. Kleemann, J. Phys. D: Appl. Phys. 42, 013001 (2009).

${ }^{11}$ P. E. Jönsson, Adv. Chem. Phys. 128, 191 (2004).

${ }^{12}$ M. Bandyopadhyay and S. Dattagupta, Phys. Rev. B 74, 214410 (2006).

${ }^{13}$ A. Rostamnejadi, H. Salamati, P. Kameli, and H. Ahmadvand, J. Magn. Magn. Mater. 321, 3126 (2009).

${ }^{14}$ J. Dormann, L. Bessais, and D. Fiorani, J. Phys. C: Solid State Phys. 21, 2015 (1988).

${ }^{15}$ S. Zhu, J. Yuan, B. Zhu, F. Zhang, B. Xu, L. Cao, X. Qiu, B. Zhao, and P. Zhang, Appl. Phys. Lett. 90, 112502 (2007).

${ }^{16}$ M. Knobel, W. C. Nunes, L. M. Socolovsky, E. D. Biasi, J. M. Vargas, and J. C. Denardin, J. Nanosci. Nanotechnol. 8, 2836 (2008).

${ }^{17}$ J. M. D. Coey, M. Viret, and S. Von Molnar, Adv. Phys. 58, 571 (2009).

${ }^{18}$ A. Rostamnejadi, M. Venkatesan, P. Kameli, H. Salamati, and J. M. D. Coey, J. Magn. Magn. Mater. 323, 2214 (2011).

${ }^{19}$ A. Rashid and S. Manzoor, J. Magn. Magn. Mater. 420, 232 (2016).

${ }^{20}$ D. Manh, P. Phong, P. Nam, D. Tung, N. Phuc, and I.-J. Lee, Phys. B: Condens. Matter 444, 94 (2014).

${ }^{21}$ S. Jadhav, D. Nikam, V. Khot, N. Thorat, M. Phadatare, R. Ningthoujam, A. Salunkhe, and S. Pawar, New J. Chem. 37, 3121 (2013).

${ }^{22}$ S. Manzoor, A. Ahmed, A. ur Rashid, S. Ahmad, and S. Shaheen, IEEE Trans. Magn. 49, 3504 (2013).
${ }^{23}$ F. Fonseca, G. Goya, R. Jardim, R. Muccillo, N. Carreno, E. Longo, and E. Leite, Phys. Rev. B 66, 104406 (2002).

${ }^{24}$ G. Goya, T. Berquo, F. Fonseca, and M. Morales, J. Appl. Phys. 94, 3520 (2003).

${ }^{25}$ P. Dey and T. Nath, Appl. Phys. Lett. 87, 162501 (2005).

${ }^{26}$ S. Dong, R. Yu, S. Yunoki, J. M. Liu, and E. Dagotto, Phys. Rev. B 78, 064414 (2008).

${ }^{27}$ S. Dong, F. Gao, Z. Wang, J. M. Liu, and Z. Ren, Appl. Phys. Lett. 90, 082508 (2007).

${ }^{28}$ T. Zhu, B. Shen, J. Sun, H. Zhao, and W. Zhan, Appl. Phys. Lett. 78(24), 3863 (2001)

${ }^{29}$ A. Rostamnejadi, M. Venkatesan, P. Kameli, H. Salamati, and J. M. D. Coey, J. Appl. Phys. 116, 043913 (2014).

${ }^{30}$ J. M. D. Coey, Magnetism and Magnetic Materials (Cambridge University Press, 2010)

${ }^{31}$ J. Nogués, J. Sort, V. Langlais, V. Skumryev, S. Suriñach, J. S. Muñoz, and M. D. Baró, Phys. Rep. 422, 65 (2005).

${ }^{32}$ Y. Sun, M. Salamon, K. Garnier, and R. Averback, Phys. Rev. Lett. 91, 167206 (2003).

${ }^{33}$ G. Tsoi, L. Wenger, U. Senaratne, R. Tackett, E. Buc, R. Naik, P. Vaishnava, and V. Naik, Phys. Rev. B 72, 014445 (2005).

${ }^{34}$ T. Zhang, X. Li, X. Wang, Q. Fang, and M. Dressel, Euro. Phys. J. B 74(3), 309 (2010). 